INPLASY

PROTOCOL

To cite: Deng et al. Different acupotomy for Stenosing tenosynovitis A protocol for systematic review and network meta-analysis. Inplasy protocol 202150059. doi:

10.37766/inplasy2021.5.0059

Received: 16 May 2021

Published: 16 May 2021

Corresponding author:

Wei Xiong

2289527835@qq.com

Author Affiliation:

Nanchang Hongdu Hospital of

Traditional Chinese Medicine

Support: None.

Review Stage at time of this submission: Data analysis.

Conflicts of interest:

None declared.

\section{Different acupotomy for Stenosing tenosynovitis A protocol for systematic review and network meta-analysis}

Deng, X1; Li, B2; Hou, X³; Xu, X4; Xiong, W5.

Review question / Objective: Stenosing tenosynovitis is a chronic aseptic inflammation caused by mechanical friction. The main clinical manifestations are local pain and limited activity of the affected parts, which reduce people's quality of life. The clinical effect of acupotomy in the treatment of Stenosing tenosynovitis is significant, and the operation is simple and the side effect is small. But there are many kinds of acupotomology, and there is a lack of comparative study between different Acupotomology. In this study, the effectiveness of four commonly used needle knife therapies (v-knife, oblique knife, crochet knife, flat knife) was ranked by the method of network meta.

Condition being studied: At present, there are many clinical reports on the treatment of STS with Acupotomy, and the clinical effect is more significant. However, there are many kinds of acupotomy, and the treatment advantages are not the same. There is a lack of comparative study between different Acupotomy treatments, which brings trouble to the choice of clinicians.In order to provide evidence-based medical evidence for the clinical selection of the best needle knife treatment for STS, the effectiveness of four commonly used needle knife therapies (v-knife, oblique knife, crochet knife and flat knife) was ranked by the method of mesh meta.

INPLASY registration number: This protocol was registered with the International Platform of Registered Systematic Review and Meta-Analysis Protocols (INPLASY) on 16 May 2021 and was last updated on 16 May 2021 (registration number INPLASY202150059).

\section{INTRODUCTION}

Review question / Objective: Stenosing tenosynovitis is a chronic aseptic inflammation caused by mechanical friction. The main clinical manifestations are local pain and limited activity of the affected parts, which reduce people's quality of life. The clinical effect of acupotomy in the treatment of Stenosing tenosynovitis is significant, and the operation is simple and the side effect is 
small. But there are many kinds of acupotomology, and there is a lack of comparative study between different Acupotomology. In this study, the effectiveness of four commonly used needle knife therapies (v-knife, oblique knife, crochet knife, flat knife) was ranked by the method of network meta.

Condition being studied: At present, there are many clinical reports on the treatment of STS with Acupotomy, and the clinical effect is more significant. However, there are many kinds of acupotomy, and the treatment advantages are not the same. There is a lack of comparative study between different Acupotomy treatments, which brings trouble to the choice of clinicians.In order to provide evidencebased medical evidence for the clinical selection of the best needle knife treatment for STS, the effectiveness of four commonly used needle knife therapies (vknife, oblique knife, crochet knife and flat knife) was ranked by the method of mesh meta.

\section{METHODS}

Participant or population: The diagnosis of STS has a clear and accepted diagnostic criteria and therapeutic criteria; There are no restrictions on age, race, gender and source of cases.

Intervention: The treatment group was only treated with Acupotomy (including v-knife, oblique knife, crochet knife and flat knife).

Comparator: While the control group was treated with closed therapy, conventional surgery and flat knife (both the treatment group and the control group could cooperate with the treatment of basic diseases of internal medicine).

Study designs to be included: There are many improved needle knife therapies in China, with higher clinical effectiveness and safety. However, there is a lack of comparison between different knives, so it is difficult to provide guidance for clinicians.Network meta-analysis can provide the best treatment plan for clinic by quantifying the treatment effect of various needle knife therapies.

Eligibility criteria: 1.Non randomized controlled trials, such as systematic reviews, reviews, animal experiments, etc. 2.Using non-standard grouping methods, such as coin tossing, odd and even numbers or patients willing to group, etc. 3.There is no definite diagnostic or therapeutic criteria. 4.The experimental group and the control group contained other interference therapy. 5.The latest one was selected for the repeated articles published in Chinese and English journals. 6.Data or full-text literature cannot be obtained.

Information sources: Randomized controlled trials (RCTs) of needle knife in the treatment of STS were searched in CNKI, Wan-Fang data, CBM, VIP, PubMed and Cochrane Library.

Main outcome(s): Cure rate, total effective rate (effective rate $=([$ Cured + markedly effective + effective]/total number of cases $\times 100 \%)$, complications, recurrence rate.

Quality assessment / Risk of bias analysis: Two reviewers used the Cochrane Handbook of systematic review to evaluate the quality of the included articles and assess the risk of bias, including selection bias, implementation bias, measurement bias, follow-up bias, reporting bias and other source bias. The results of the evaluation are "high", "risk", "low risk" and "unclear risk"

Strategy of data synthesis: Revman $\mathbf{5 . 3}$ software was used to assess the risk of bias. Stata 14.2 and gemtc $\mathbf{0 . 1 4}$.3 software were used to conduct network metaanalysis using Markov chain Monte Carlo (MCMC). Stata was used to calculate the Sucra (surface under the cumulative ranking curves, Sucra) value and area under the curve, so as to rank the efficacy of various interventions. When there is a closed loop in the network diagram, the inconsistency test is needed. If there is no obvious inconsistency between the two, Bayesian inference is performed in the 
consistency model, and the convergence of the results is evaluated by the potential scale reduced factor (psrf). When $1.00 \leq$ psrf $\leq 1.05$, the convergence of the results is good.

Subgroup analysis: According to the different sources of heterogeneity, subgroup analysis can be carried out, such as treatment time, course of disease, underlying disease, race, gender, age, etc.

Sensitivity analysis: Observe the heterogeneity of different experiments, observe whether the combined results change after different treatments, and analyze the strength, reliability and stability of the results.

Country(ies) involved: China.

Keywords: Stenosing tenosynovitis; needle knife; network meta-analysis; protocol.

Contributions of each author:

Author 1 - Xiaoling Deng.

Author 2 - Bingru Li.

Author 3 - Xinju Hou.

Author 4 - Xing Xu.

Author 5 - Wei Xiong. 